\section{For Patients Needing Oral Anticoagulation for Atrial Fibrillation and Dual Antiplatelet Therapy after Percutaneous Coronary Intervention, Is Double Therapy Preferred over Triple Therapy?}

\section{THE "PRO" SIDE}

For many years clinicians have faced a conundrum in managing patients who require both oral anticoagulation and dual antiplatelet therapy after percutaneous coronary intervention (PCI). This scenario is commonly encountered in practice, given that approximately $20 \%$ of patients with atrial fibrillation will require PCI at some time, and up to $21 \%$ of patients with acute coronary syndrome (ACS) will also have new or established atrial fibrillation. ${ }^{1,2}$ The need for triple therapy - that is, the use of an oral anticoagulant and dual antiplatelet therapy - has not been studied with rigour but has been adopted in practice, as there have been no perceived alternatives. However, cohort studies have shown that triple therapy leads to an increased risk of major bleeds. ${ }^{3}$ We argue that there is now adequate evidence to avoid triple therapy and to change the standard of care for this population to double therapy, that is, the use of an anticoagulant (preferably a direct-acting oral anticoagulant) and a single antiplatelet agent (preferably a P2Y12 inhibitor).

The first study to investigate the use of double therapy was the WOEST trial (What Is the Optimal Antiplatelet and Anticoagulant Therapy in Patients with Oral Anticoagulation and Coronary Stenting), published in $2013 .{ }^{4}$ In this trial, patients $(n=573)$ who were receiving vitamin $\mathrm{K}$ antagonists and who underwent PCI were randomly assigned to receive acetylsalicylic acid (ASA) and clopidogrel (i.e., triple therapy) or clopidogrel alone (i.e., double therapy). At 1 year, the rate of major bleeding was $19.4 \%$ among those receiving double therapy and $44.4 \%$ for the triple-therapy group (hazard ratio $[\mathrm{HR}] 0.35$, 95\% confidence interval [CI] 0.25-0.50, $p<0.0001)$. With omission of the ASA there was no signal for loss of efficacy, given that the secondary combined end point of death, myocardial infarction, stroke, target vessel revascularization, and stent thrombosis was lower in the double-therapy group than in the triple-therapy group (11.1\% versus $17.6 \%$, adjusted HR 0.56 , 95\% CI 0.35-0.91). Of note, although the majority of patients (69\%) were using anticoagulation for atrial fibrillation, patients with other indications (e.g., mechanical valve) were also included in this study.

The use of direct-acting oral anticoagulants in a double-therapy regimen was studied in a randomized controlled fashion in the PIONEER AF-PCI trial (Open-Label, Randomized, Controlled, Multicentre Study Exploring Two Treatment Strategies of Rivaroxaban and a Dose-Adjusted Oral Vitamin K Antagonist Treatment Strategy in Subjects with Atrial Fibrillation who Undergo
Percutaneous Coronary Intervention). ${ }^{5}$ Patients $(n=2124)$ with atrial fibrillation who underwent PCI were randomly assigned, within 3 days of the procedure, to receive 1 of the following 3 regimens: rivaroxaban $15 \mathrm{mg}$ daily (or $10 \mathrm{mg}$ daily if creatinine clearance was less than $50 \mathrm{~mL} / \mathrm{min}$ ) plus P2Y12 inhibitor; rivaroxaban $2.5 \mathrm{mg}$ BID and dual antiplatelet therapy (for 1, 6, or 12 months); or triple therapy with warfarin. The primary outcome-bleeding that was clinically significant or required medical attention-was lower in both rivaroxaban groups than in the warfarin triple-therapy group (rivaroxaban $15 \mathrm{mg}$ daily plus P2Y12 inhibitor: $16.8 \%$ versus $26.1 \%$, HR 0.59, 95\% CI 0.47-0.76, $p<0.001$; rivaroxaban $2.5 \mathrm{mg}$ BID plus dual antiplatelet therapy, $18.0 \%$ versus $26.7 \%$, HR $0.63,95 \%$ CI $0.50-0.80, p<0.001)$. The rate of myocardial infarction, stroke, or death from cardiovascular causes was similar across all groups. ${ }^{5}$

Next, the RE-DUAL PCI trial (Randomized Evaluation of Dual Antithrombotic Therapy with Dabigatran versus Triple Therapy with Warfarin in Patients with Nonvalvular Atrial Fibrillation Undergoing Percutaneous Coronary Intervention) randomly assigned patients ( $n=2725)$ with atrial fibrillation within 5 days after PCI to receive either triple therapy with warfarin, a P2Y12 inhibitor, and ASA (with ASA being discontinued at 1 month for patients with bare metal stents or at 3 months for those with drug-eluting stents) or double therapy with dabigatran (110 or $150 \mathrm{mg}$ BID) and P2Y12 inhibitor (clopidogrel or ticagrelor). ${ }^{6}$ The primary end point (major or clinically relevant non-major bleeding) was significantly lower in the dualtherapy groups receiving dabigatran $150 \mathrm{mg}$ BID (20.2\% versus $25.7 \%$, HR 0.72 , 95\% CI $0.58-0.88, p<0.001)$ or dabigatran $110 \mathrm{mg}$ BID (15.4\% versus $26.9 \%$, HR 0.52 , 95\% CI 0.42-0.63, $p<0.001$ ). Although the study was not sufficiently powered for the composite efficacy end point of thromboembolic events, death, or unplanned revascularization, the pooled double-therapy groups met criteria for non-inferiority to triple therapy $(13.7 \%$ versus $13.4 \%$, HR 1.04, 95\% CI 0.85-1.29, $p=0.005){ }^{6}$

The latest trial in search of the ideal antithrombotic therapy was published in early 2019. The AUGUSTUS trial (Open-label, $2 \times 2$ Factorial, Randomized Controlled, Clinical Trial to Evaluate the Safety of Apixaban vs. Vitamin KAntagonist and Aspirin vs. Aspirin Placebo in Patients with Atrial Fibrillation and Acute Coronary Syndrome or Percutaneous Coronary Intervention) applied randomization to patients $(n=4614)$ with atrial fibrillation and an indication for dual antiplatelet therapy (ACS or PCI). Using a $2 \times 2$ factorial design, the trial examined 2 hypotheses, one related to the safety and efficacy of warfarin compared with apixaban and the other related to the safety and efficacy of low-dose ASA compared with placebo. ${ }^{7}$ All patients received a P2Y12 inhibitor. Randomization had to occur within 14 days of ACS or PCI. This trial demonstrated that triple therapy with either warfarin or apixaban was linked to higher rates of bleeding than was the case with dual therapy (16.1\% versus $9.0 \%$, 
HR 1.90, 95\% CI 1.59-2.24, $p<0.001)$. Lower rates of bleeding (major or clinically relevant non-major) were seen in the apixaban group than in the warfarin group (10.5\% versus $14.7 \%$, HR 0.69 , 95\% CI $0.58-0.81, p<0.001)$. As with the previous trials, AUGUSTUS was not sufficiently powered to evaluate efficacy outcomes. However, the incidence of death or hospital admission was lower among patients taking apixaban than among those taking warfarin, and the incidence of death, hospital admission, and ischemic events was similar among patients receiving double versus triple therapy ${ }^{7}$

A network meta-analysis, which analyzed the WOEST, RE-DUAL, PIONEER-PCI-AF, and AUGUSTUS trials and which pooled 10026 patients for analysis, has now been published, and it begins to address the power concerns with the individual trials. ${ }^{8}$ Its conclusion re-emphasized that omitting ASA lowers the rate of major bleeding without a significant change in major adverse cardiac events, relative to triple-therapy regimens. ${ }^{8}$ We acknowledge that there are limitations to the trials discussed, especially the fact that all trials used safety as a primary end point, and we also acknowledge that they were underpowered with respect to efficacy outcomes of cardiovascular death, myocardial infarction, and stent thrombosis. However, it is unlikely that there will ever be a randomized controlled trial with a primary efficacy outcome, given sample size requirements of at least 20000 participants. In addition, although we have been using the term "double therapy", patients in these studies received triple therapy for some time before randomization (up to 3 days in the PIONEERAF PCI trial, up to 5 days in the RE-DUAL trial, and up to 14 days in the AUGUSTUS trial). ${ }^{5-7}$ Finally, physicians recruiting patients for these trials may not have approached individuals with high thrombotic risk (such as left main artery stenting or high thrombotic burden) to discuss study involvement. Such selective recruitment, a common problem in trials, would limit the generalizability of study findings to those with low to moderate thrombotic risk.

With the evidence available today, triple therapy as a blanket approach for all patients leads to an unnecessarily high rate of bleeding with no obvious benefit with respect to efficacy. For the population at large, available evidence points clinicians to double therapy, with traditional triple therapy being reserved for the outliers of the population who are at above-normal risk of thrombotic complications.

\section{References}

1. Schmitt J, Duray G, Gersh BJ, Hohnloser SH. Atrial fibrillation in acute myocardial infarction: a systematic review of the incidence, clinical features and prognostic implications. Eur Heart J. 2009;30(9):1038-45.

2. Kralev S, Schneider K, Lang S, Suselbeck T, Borggrefe M. Incidence and severity of coronary artery disease in patients with atrial fibrillation undergoing first-time coronary angiography. PloS One. 2011;6(9):e24964.

3. Hansen ML, Sorensen R, Clausen MT, Fog-Petersen ML, Raunso J, Gadsboll N, et al. Risk of bleeding with single, dual, or triple therapy with warfarin, aspirin, and clopidogrel in patients with atrial fibrillation. Arch Intern Med. 2010;170(16):1433-41.

4. Dewilde WJ, Oirbans T, Verheugt FW, Kelder JC, De Smet BJ, Herrman JP, et al. Use of clopidogrel with or without aspirin in patients taking oral anticoagulant therapy and undergoing percutaneous coronary intervention: an open-label, randomised, controlled trial. Lancet. 2013;381(9872):1107-15.

5. Gibson CM, Mehran R, Bode C, Halperin J, Verheugt FW, Wildgoose P, et al. Prevention of bleeding in patients with atrial fibrillation undergoing PCI. N Engl J Med. 2016;375(25):2423-34.
6. Cannon CP, Bhatt DL, Oldgren J, Lip GYH, Ellis SG, Kimura T, et al. Dual antithrombotic therapy with dabigatran after PCI in atrial fibrillation. $N$ Engl JMed. 2017;377(16):1513-24.

7. Lopes RD, Heizer G, Aronson R, Vora AN, Massaro T, Mehran R, et al. Antithrombotic therapy after acute coronary syndrome or PCI in atrial fibrillation. N Engl J Med. 2019;380(16):1509-24.

8. Lopes RD, Hong H, Harskamp RE, Bhatt DL, Mehran R, Cannon CP, et al Safety and efficacy of antithrombotic strategies in patients with atrial fibrillation undergoing percutaneous coronary intervention: a network meta-analysis of randomized controlled trials. JAMA Cardiol. 2019;4(8):747-55.

Lindsay Dryden, BMSc, BScPhm, PharmD, ACPR

Heather Kertland, BScPhm, PharmD, FCSHP

St Michael's Hospital

Toronto, Ontario

Competing interests: Heather Kertland has received personal fees from BMS/Pfizer Alliance for activities outside the scope of this article. No other competing interests were declared.

\section{THE "CON" SIDE}

Dual antiplatelet therapy, consisting of acetylsalicylic acid (ASA) with a P2Y12 antagonist, is required after percutaneous coronary intervention (PCI) or acute coronary syndrome (ACS) to prevent stent thrombosis and myocardial infarction. A common scenario arises when a patient needs dual antiplatelet therapy after PCI and also oral anticoagulation for atrial fibrillation. The use of dual antiplatelet therapy plus oral anticoagulation (known as triple therapy) carries concerns about increased risk of hemorrhage. Thus, clinicians are faced with a dilemma: either treat post-PCI patients who have atrial fibrillation with triple therapy to reduce the risk of cardioembolic and ischemic events, with acceptance of a higher risk of bleeding, or reduce the antithrombotic regimen to minimize the risk of bleeding, with acceptance of the possibility of more ischemic events. Both bleeding and cardiovascular events (stroke, stent thrombosis, myocardial infarction) are associated with poor outcomes. ${ }^{1}$

In recent years, a flurry of large trials have been published that attempt to provide guidance in this clinical dilemma. The WOEST trial was the first study to investigate triple therapy versus double therapy consisting of clopidogrel plus warfarin ${ }^{2}$ (Table 1). Subsequent trials-PIONEER AF-PCI, RE-DUAL PCI, and AUGUSTUS - compared use of a P2Y12 antagonist plus direct oral anticoagulant with triple therapy. ${ }^{3-5}$ On the basis of these trials, the approach of omitting ASA and instead using only a P2Y12 antagonist (mainly clopidogrel) plus oral anticoagulant (i.e., double therapy) for post-PCI patients who have atrial fibrillation has been rapidly adopted. However, a review of the evidence, as outlined below, indicates that we should not universally omit ASA and employ double therapy for all post-PCI patients with atrial fibrillation. 


\section{Trials of Double Therapy Were Safety Trials with Major Bleeding as a Primary End Point}

Foremost, the trials evaluating double therapy (WOEST, PIONEER AF-PCI, RE-DUAL, AUGUSTUS) were designed as safety trials. As such, the incidence of bleeding was the primary end point for the comparison between triple therapy and double therapy; cardiovascular outcomes were secondary composite end points. In all of these trials, bleeding was significantly lower in patients treated with double therapy than among those treated with triple therapy. Thus, the trials demonstrated the intuitive conclusion that the less antithrombotic therapy a patient receives, the lower the patient's risk of bleeding. Although there was no difference in stent thrombosis or myocardial infarction between the 2 groups across these trials, the trials were underpowered to detect such cardiovascular end points. The authors of the PIONEER AF-PCI trial $(n=2124)$ estimated that a study to establish superiority of clopidogrel plus rivaroxaban over triple therapy in terms of myocardial infarction would require more than 40000 patients. $^{3}$ The RE-DUAL trial was originally designed to enrol 8520 patients to allow for evaluation of an efficacy end point of thrombotic events, but because of feasibility issues, only 2725 patients were recruited, leaving it underpowered. ${ }^{4}$ The AUGUSTUS trial, ${ }^{5}$ which evaluated clopidogrel plus oral anticoagulant versus ASA plus clopidogrel plus oral anticoagulant, demonstrated lower rates of major or clinically relevant non-major bleeding in the double-therapy group and no statistical difference in the rates of ischemic events. However, the statistical framework for AUGUSTUS, including sample size calculation, was designed for comparing apixaban and warfarin, not for comparing ASA and placebo. ${ }^{6}$ Of concern, the authors of the AUGUSTUS study noted a greater number of coronary ischemic events among patients who did not take ASA relative to those who did take ASA (ASA versus placebo, hazard ratio [HR] for death or ischemic event $0.89,95 \%$ confidence interval [CI] 0.71-1.11; HR for myocardial infarction $0.81,95 \%$ CI $0.59-1.12$; $\mathrm{HR}$ for stent thrombosis $0.52,95 \%$ CI $0.25-1.08)$. Although this observation was not statistically significant, the authors stated that it should be considered exploratory and noted that a similar pattern of more coronary ischemic events with omission of ASA had been observed in similar trials. ${ }^{5}$ Thus, the conclusion from these often-quoted trials is that omitting ASA and using double therapy leads to less major bleeding. However, we cannot definitively state that the rates of ischemic events are unchanged with double therapy, given that all of the trials were underpowered to detect these important clinical events, heterogeneity existed among the trials, and the studies included mainly patients with lower ischemic risk.

\section{Dual Antiplatelet Therapy Has Decades of Highest-Level Evidence}

Dual antiplatelet therapy after PCI is supported by decades of literature and the highest level of evidence. ${ }^{7,8}$ There is also robust evidence showing superior reduction in ischemic events with longer-term dual antiplatelet therapy after PCI (the PEGASUS trial). ${ }^{9,10}$

The use of warfarin or direct oral anticoagulant provides little benefit in post-PCI patients in terms of preventing stent thrombosis or recurrent myocardial infarction. ${ }^{11}$ In fact, there was

Table 1. Summary of Major Trials* Comparing Triple Therapy and Double Therapy

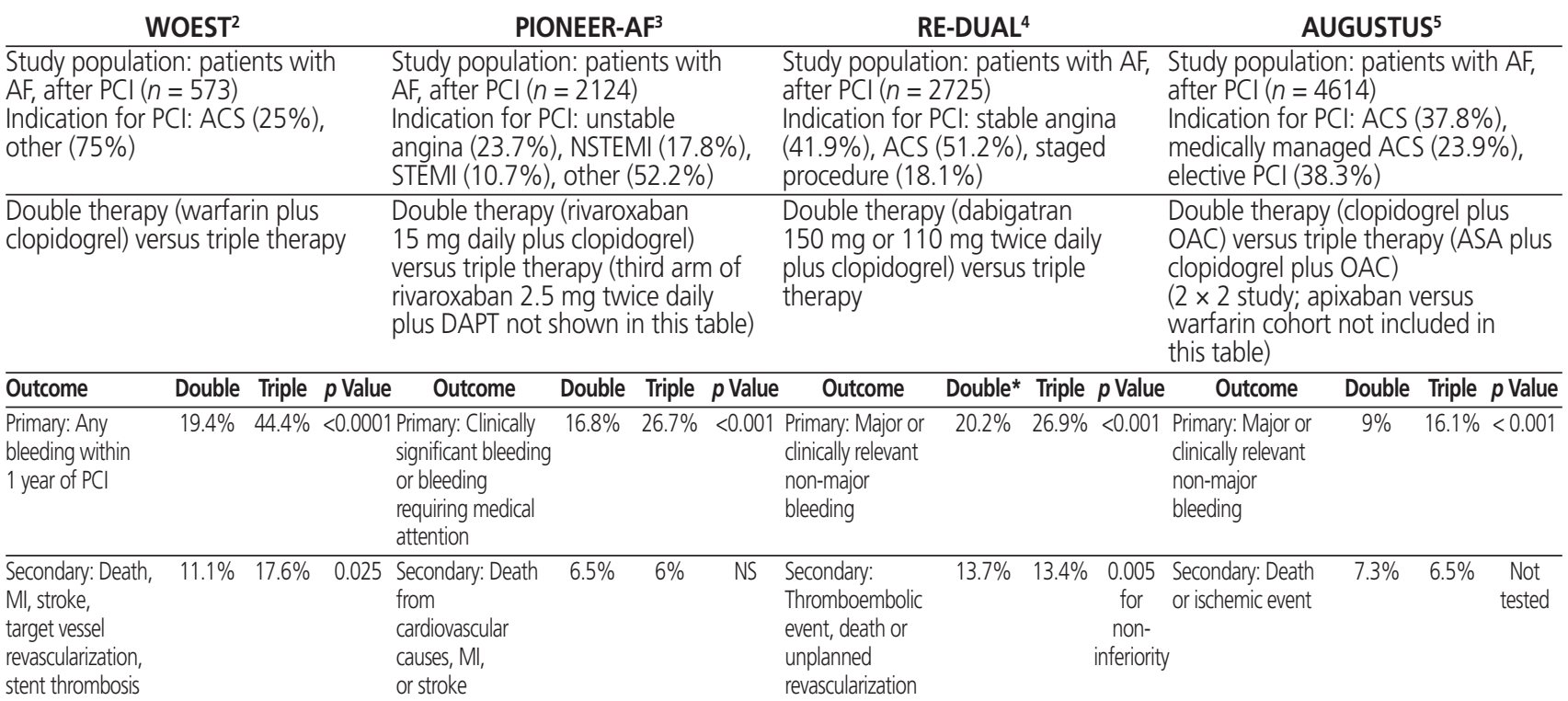

$\overline{\mathrm{ACS}}=$ acute coronary syndrome, $\mathrm{AF}=$ atrial fibrillation, $\mathrm{ASA}=$ acetylsalicylic acid, DAPT = dual antiplatelet therapy, Ml = myocardial infarction, $\mathrm{NS}=$ not significant, NSTEMI = non-ST elevation myocardial infarction, $\mathrm{OAC}=$ oral anticoagulant, $\mathrm{PCI}=$ percutaneous coronary intervention, STEMI = ST elevation myocardial infarction.

*Dabigatran 150 mg bid. 
a signal within the RE-LY trial that dabigatran might actually increase rates of myocardial infarction. ${ }^{12}$ However, re-analysis of data from RE-LY and subsequent trials did not replicate this finding, and it has now been established that use of direct oral anticoagulant does not influence rates of coronary ischemic events. ${ }^{13,14}$ Thus, we can conclude that oral anticoagulant does not contribute to reducing stent thrombosis or myocardial infarction; however, dual antiplatelet therapy is well proven in preventing these coronary events. We should not be distracted by recent trials of double therapy to forget the vast amount of prior evidence supporting the critical role of ASA as part of dual antiplatelet therapy in the context of triple therapy.

\section{International Cardiology Guidelines Still Endorse Triple Therapy after $\mathrm{PCl}$ in Patients with Atrial Fibrillation}

Canadian, US, and European guidelines all advocate that the choice between triple therapy and double therapy should be based on the balance between thrombotic risk and bleeding risk for each patient. ${ }^{15-17}$ If the patient's thrombotic risk is high and bleeding risk is low, then triple therapy is recommended. If the patient's thrombotic risk is low and bleeding risk is high, then double therapy should be considered. The most recent Canadian Cardiovascular Society guidelines on antiplatelet therapy state that the timing of discontinuation of ASA will vary depending on the individual patient's ischemic and bleeding risk. ${ }^{15}$ The duration of triple therapy can be individualized, but ASA as part of dual antiplatelet therapy in the context of triple therapy has a prominent role in the first months after stent insertion and in high-risk patients.

\section{Conclusion}

Omitting ASA and using double therapy in patients with atrial fibrillation after PCI should not be the default regimen. The decision to continue or discontinue ASA should be based on assessment of the individual patient's thrombotic and bleeding risk. The quantity and quality of evidence supporting use of ASA as part of dual antiplatelet therapy in the context of triple therapy to reduce coronary events, particularly in patients with high ischemic risk and low bleeding risk, outweighs the evidence for double therapy.

\section{References}

1. Steg PG, Huber K, Andreotti F, Arnesen H, Atar D, Baadimon L, et al. Bleeding in acute coronary syndromes and percutaneous coronary interventions: position paper by the Working Group on Thrombosis of the European Society of Cardiology. Eur Heart J. 2011;32(15):1854-64.

2. Dewilde JW, Oirbans T, Verheugt FW, Kelder JC, De Smet BJ, Herrman JP, et al. Use of clopidogrel with or without aspirin in patients taking oral anticoagulant therapy and undergoing percutaneous coronary intervention: an open-label, randomised, controlled trial. Lancet. 2013;381(9872): 1107-15.
3. Gibson CM, Mehran R, Bode C, Halperin J, Verheugt FW, Wildgoose $\mathrm{P}$, et al. Prevention of bleeding in patients with atrial fibrillation undergoing PCI. N Engl J Med. 2016;375(25):2423-34.

4. Cannon CP, Bhatt DL, Oldgren J, Lip GYH, Ellis SG, Kimura T, et al. Dual antithrombotic therapy with dabigatran after PCI in atrial fibrillation. N Engl J Med. 2017;377(16):1513-24.

5. Lopes RD, Heizer G, Aronson R, Vora AN, Massaro T, Mehran R, et al Antithrombotic therapy after acute coronary syndrome or PCI in atrial fibrillation. $N$ Engl J Med. 2019;380(16):1509-24.

6. Lopes RD, Vora AN, Liaw D, Granger CB, Darius H, Goodman SG, et al. An open-label, $2 \times 2$ factorial, randomized controlled trial to evaluate the safety of apixaban vs. vitamin $\mathrm{K}$ antagonist and aspirin vs. placebo in patients with atrial fibrillation and acute coronary syndrome and/or percutaneous coronary intervention: rationale and design of the AUGUSTUS trial. Am Heart J. 2018;200:17-23.

7. Chua D, Ignaszewski A. Clopidogrel in acute coronary syndromes. $B M J$. 2009;338:b1180.

8. Chua D, Nishi C. New antiplatelet agents for cardiovascular disease. CMAJ. 2013;185(16):1405-11.

9. Mauri L, Kereiakes DJ, Yeh RW, Driscoll-Shempp P, Cutlip DE, Streg PG, et al. Twelve or 30 months of dual antiplatelet therapy after drug-eluting stents. N Engl J Med. 2014;371(23):2155-66.

10. Bonaca MP, Bhatt DL, Cohen M, Steg PG, Storey RF, Jensen EC, et al. Long-term use of ticagrelor in patients with prior myocardial infarction. N Engl J Med. 2015;372(19):1791-800.

11. Leon MB, Balm DS, Popma JJ, Gordon PC, Cutlip DE, Ho KK, et al. A clinical trial comparing three antithrombotic drug regimens after coronary artery stenting. N Engl J Med. 1998;339(23):1665-71.

12. Cairns JA, Connolly S, McMurty S, Stephenson M, Talajic M; CCS Atrial Fibrillation Guidelines Committee. Canadian Cardiovascular Society atrial fibrillation guidelines 2010: prevention of stroke and systemic thromboembolism in atrial fibrillation and flutter. Can J Cardiol. 2011;27(1):74-90.

13. Hohnloser SH, Eikleboom JW. Direct oral anticoagulants and myocardial infarction: the dust is settling. J Am Coll Cardiol. 2018;72(1):27-8.

14. Skanes AC, Healey JS, Cairns JA, Dorian P, Gillis AM, McMurtry MS, et al. Focused 2012 update of the Canadian Cardiovascular Society atrial fibrillation guidelines: recommendations for stroke prevention and rate/rhythm control. Can J Cardiol. 2012:28(2):125-36.

15. Metha SR, Bainey KR, Cantor WJ, Lordkipanidze M, Marquis-Gravel G, Robinson SD, et al. 2018 Canadian Cardiovascular Society/Canadian Association of Interventional Cardiology focused update of the guidelines for the use of antiplatelet therapy. Can J Cardiol. 2018;34(3):214-33.

16. Valgimigli M, Bueno H, Byrne RA, Collet JP, Costa F, Jeppsson A, et al. 2017 ESC focused update on dual antiplatelet therapy in coronary artery disease developed in collaboration with EACTS: the task force for dual antiplatelet therapy in coronary artery disease of the European Society of Cardiology (ESC) and the European Association for Cardio-Thoracic Surgery (EACST). Eur Heart J. 2018;39(3):213-60.

17. January CT, Wann LS, Calkins H, Chen LY, Cigarroa JE, Cleveland JC, et al. 2019 AHA/ACC/HRS focused update of the 2014 AHA/ ACC/HRS guideline for the management of patients with atrial fibrillation. J Am Coll Cardiol. 2019;74(1):104-32.

Doson Chua, BSC(Pharm), PharmD, FCSHP, BCPS(AQ), BCCP

Clinical Pharmacy Specialist, Cardiology

St Paul's Hospital

Clinical Professor, Faculty of Pharmaceutical Sciences

University of British Columbia

Vancouver, British Columbia

Competing interests: None declared 